\title{
BMJ Open Study protocol for the use of photobiomodulation with red or infrared LED on waist circumference reduction: a randomised, double-blind clinical trial
}

\author{
Marcelo Marreira, Lidiane Rocha Mota, Daniela Fátima Teixeira Silva (1) , \\ Christiane Pavani
}

To cite: Marreira M, Rocha Mota L, Silva DFT, et al. Study protocol for the use of photobiomodulation with red or infrared LED on waist circumference reduction: a randomised, double-blind clinical trial. BMJ Open 2020;10:e036684. doi:10.1136/ bmjopen-2019-036684

- Prepublication history and additional material for this paper are available online. To view these files, please visit the journal online (http://dx.doi. org/10.1136/bmjopen-2019036684).

Received 26 December 2019 Revised 06 July 2020 Accepted 07 July 2020

D) Check for updates

(c) Author(s) (or their employer(s)) 2020. Re-use permitted under CC BY-NC. No commercial re-use. See rights and permissions. Published by BMJ.

Biophotonics Applied to Health Sciences, Universidade Nove de Julho, Sao Paulo, Brazil

Correspondence to Dr Christiane Pavani; chrispavani@gmail.com

\section{ABSTRACT}

Introduction The search for non-invasive procedures to reduce localised adiposity in aesthetics clinics has recently been increasing. In this context, procedures, such as cryolipolysis, ultracavitation, photobiomodulation (PBM) and other techniques have been proposed. Some studies have shown that PBM can be used in body contouring. However, there is no standardisation of the protocol. More than that, as in other techniques for reducing adipose tissue, the availability of triacylglycerol may affect the lipid profile in the blood, bringing consequences to the general health of an individual. This work will aim to compare the light wavelengths when using PBM as a technique for reducing the abdominal waist circumference, while also evaluating the efficacy of the method. Changes in the lipid profile in the blood, with a long-term follow-up, will also be appraised.

Methods and analysis This will be a controlled, randomised, double-blind, single-centred clinical trial. 174 patients will be recruited at the Nove de Julho University, Brazil, and then divided into three groups: Group A-RED PBM; Group B-INFRARED PBM; Group C-PLACEBO (Sham) treatment. The treatments will consist of eight sessions, two times a week, for 4 weeks. At each session, the participants will receive 30 minutes PBM (using a radiant exposure of $127 \mathrm{~J} / \mathrm{cm}^{2}$ ), with an abdominal strap containing 4 LED clusters, with 72 devices each, following the indication of randomisation. All of the groups will receive 30 min of Aussie Current, at $4 \mathrm{kHz}$, modulated at $10 \mathrm{~Hz}, 40-60 \mathrm{~mA}$. The main outcome of this study will be waist circumference reduction. The secondary variables will be anthropometric data, lipid profile, liver function and adipose tissue thickness, changes in the local microcirculation, and the quality of life and selfesteem. The analyses will be performed at four stages of the research, D0, end of the eighth session (D30), 15 days after the last session (FU15), 90 days after the last session (FU90) and 180 days after the last session (FU180).

Ethics and dissemination The Ethics Committee of the Nove de Julho University, Brazil, approved the modified version of this project under No. 3414146 on 26 June 2019. This study is not yet recruiting. The results obtained
Strengths and limitations of this study

- The use of the same dosimetry at different wavelengths will allow for a real comparison between red and infrared as being the most suitable wavelength for body contouring.

- Analyses of body contouring will be performed by non-invasive methods.

- The waist circumference measurement will not discriminate the factors underlying the volume modifications.

- The habits of the participants, such as diet and exercise routines, may affect the results.

- Gender may affect the results and be dependent on the number of participants of each gender. These differences may not be considered by the statistical analysis.

will be published in a peer-reviewed journal in the related field.

Trial registration number Brazilian Registry of Clinical Trials-ReBec (RBR-9bwxcx).

\section{INTRODUCTION}

Fat storage is intended to protect the human body in cases of prolonged fasting, intense physical activity and temperature regulation. Once freed from these situations, fat is stored unnecessarily, putting the individual at the risk of health problems, together with a greater propensity for pathologies, such as systemic arterial hypertension, diabetes mellitus, metabolic syndrome and even some types of neoplasms. ${ }^{1-3}$

Another type of negative impact that is related to excessive fat storage is body dissatisfaction. This naturally leads to a decrease in the individual's self-esteem. ${ }^{4}{ }^{5}$ Studies have shown that aesthetic treatments significantly increase a patient's quality of life. They 
are associated with improved self-esteem. ${ }^{6-9}$ There are some traditional surgical methods for the reduction of abdominal adiposity. However, the methods are invasive techniques, which may present a high number of complications, such as bruising, seroma, pain, perforated organs and viscera, as well as with an increased risk of deep vein thrombosis. ${ }^{10-13}$ The demand for minimally invasive procedures that are aimed at reducing abdominal fat has increased by about $137 \%$, while the demand for surgical procedures has decreased by around $16 \% .{ }^{14}$

Among the minimally invasive techniques, one can mention low-level laser therapy, which has recently also been called photobiomodulation (PBM). PBM has many novel advantages when compared with traditional techniques, such as surgical procedures, since it can guarantee the preservation of the noble adjacent structures, such as the nerves, the blood vessels and the skin. ${ }^{15}$ PBM has been widely studied for several applications, due to its important biochemical cellular consequences and its few side effects. ${ }^{1617}$ Some manuscripts have described erythema and oedema as the main side effects of PBM, but importantly, these side effects may have been higher as a result of the patient using any drug that increased photosensitivity.

Some other studies have shown that PBM can be used in body contouring. ${ }^{18-22}$ Sadly, there is no standardisation of the protocol. The treatments vary in terms of the number of sessions (6-28), their frequency (1-3 times per week) and wavelength (532 nm, $635 \mathrm{~nm}, 660$ $\mathrm{nm}, 1060 \mathrm{~nm}$ ), while other dosimetry information, such as irradiance $\left(\mathrm{W} / \mathrm{cm}^{2}\right)$ and radiant exposure $\left(\mathrm{J} / \mathrm{cm}^{2}\right)$, are frequently not mentioned. Recently, Croghan and coworkers showed that two times a week was the best frequency when compared with one or three times a week. This was in terms of improving the patients' quality of life and body satisfaction, as well as their weight, waist circumference, body mass index (BMI) and body fat mass reduction. ${ }^{18}$ However, more studies are needed, in order to standardise the wavelength, the dosimetry and the application time, as well as the durability of the results achieved.

One of the proposed mechanisms for a PBM effect in adipose tissue is the formation of transient pores in the adipocyte membranes, thus allowing for the lipids to escape. ${ }^{15}$ Adipocyte apoptosis activation has also been proposed. The production of reactive oxygen species is also possible, due to the action of PBM, and this is related to the mitochondrial activation, on account of the radiation absorption by the cytochrome $\mathrm{c}$ oxidase molecules. This is followed by an increased ATP synthesis and with an increased cyclic adenosine monophosphate messenger, which can trigger the activation of the lipases that perform the hydrolysis of the triglycerides into fatty acids and glycerol. ${ }^{23}$

Some reports have affirmed that the results obtained by the use of PBM for reducing waist circumference are modest and that the reduction is temporary, which deserves much greater attention from researchers for a better understanding of this factor. These effects may be associated with the mechanisms of action and the dosimetric parameters being used. ${ }^{24}$ When taken to the tissues, the free fatty acids are used as an energy source during beta-oxidation for the production of ATP. In some literature reports, PBM is associated with aerobic or resistance exercise, while other reports have mentioned waist and arm circumference reductions with the use of PBM, displaying an absence of diet restrictions or exercise requirements. ${ }^{25-29} \mathrm{It}$ is also reported that the amount of fat mobilised during a PBM session is similar to the amount that is consumed during a meal, in such a way that it can be absorbed by normal body energy requirements (and/or exercise routine), while at the same time, the risk of atherosclerosis is not increased by the treatment. ${ }^{30}$ On the other hand, if not consumed, these fatty acids may be re-esterified and redistributed throughout the body, ${ }^{30}$ causing no final changes in waist circumference. Since neuromuscular electrical stimulation increases energy expenditure in a similar way to that associated with exercise, the protocol will be complemented with the Aussie current application. ${ }^{31}$

As for other techniques for reducing the adipose tissue, the availability of triacylglycerol may affect the lipid profile in the blood, bringing consequences for the general health of the individual. Some studies have shown that these important treatments may affect the serum lipid levels, while others affirm that there are no changes in the serum lipid levels. ${ }^{32}$ Given these scenarios, this work will aim to compare the different light wavelengths when using PBM as a technique for the reduction of abdominal waist circumference, while at the same time, evaluating the efficacy of the method, and by following the changes in the lipid profile in the blood, as well as with reviewing the long-term follow-up.

\section{METHODS}

\section{Study design}

This will be a controlled, randomised, double-blind, single-centred clinical trial, designed in accordance with the criteria as established by the Standard Protocol Items: Recommendations for Interventional Trials. It will be conducted at the Nove de Julho University, located in the city of São Paulo, Brazil. The recruitment will be performed from September to November 2020 through the university website. Thus, the selection of sites includes urban locations, the city of São Paulo and its neighbourhoods. After verbal and written explanations regarding the procedures, the risks and the benefits by MM (a coauthor of this protocol), those individuals who agree to participate in the study will sign an informed consent form. Based on an anamnesis questionnaire, the researchers will check if the participants meet the inclusion/exclusion criteria. The anamnesis questionnaire will include identification data, anthropometric data, clinical history and daily living habits, especially dietary intake, physical activity assessments and menstrual period appraisals. 
Since dietary intake and physical activity may have direct effects on the results, at each evaluation (before the treatment and the follow-up sessions), 3 days of food records and physical activity levels will be measured. The enrolment period will be extended until the sample size is reached.

\section{Patient and public involvement statement}

The patients and/or the public will not be involved in the design, the recruitment or in the conduct of the study.

\section{Inclusion criteria}

This study will include men and women aged 25-45 years, with a BMI of between 18 and $29.9 \mathrm{~kg} / \mathrm{m}^{2}$ (normotrophic and overweight), together with hyperplasia of the abdominal fat tissue (abdominal skin folds higher than $20 \mathrm{~mm}$ ). Those who agree to participate in this research will sign an informed consent form (see online supplementary file 1).

\section{Exclusion criteria}

The following people will be excluded from this survey: those participants who are undergoing aesthetic treatments to reduce waist circumference; those who have been previously submitted to abdominoplasty or liposuction surgeries; those who are on a diet, in order to reduce their measurements; those people who engage in a physical activity more than two times a week; those who are using or have taken drugs or food supplements in last 90 days, in order to reduce their measurements and their weight, which may affect their lipid metabolism, appetite or nutrients absorption; those who have been submitted previously to oophorectomy; those with signs and/or symptoms of climacteric at the menopause; pregnant or lactating women; those participants who are not regular in attending the sessions; those participants who present metabolic dysfunctions (diabetes and thyroid disorders), cardiovascular problems (hypertension, cardiac insufficiency, arrhythmia, thrombosis, pacemaker use), respiratory issues (asthma, chronic obstructive pulmonary disease), haematological disturbances (anaemia), renal, non-alcoholic fatty liver disease, dermatological or digestive disorders (gastritis, ulcers); those with a history of oncological pathology; those with cognitive deficits.

\section{Sample calculation}

In order to calculate the sample size, a study showing the therapeutical effects of PBM when associated with aerobic plus resistance training was used. ${ }^{26}$ The researchers used the highest and the lowest abdominal circumference values, as well as the SD of the measurements. The highest and lowest abdominal circumference values for the PBM group were 107.687 and 103.812, respectively, and the highest SD of the measurements was 10.024, with 3 being the number of intervention groups in the study. The effect size, hence, was calculated when using these values, as described below:

$$
\Delta=\frac{\text { bigger }- \text { smaller }}{\left(\frac{\sigma}{\sqrt{n}}\right)^{2}}=\frac{107.687-103.812}{\left(\frac{10.024}{\sqrt{3}}\right)^{2}}=0.116
$$

When using the effect size value as calculated above, the sample size was calculated using $G^{*}$ Power software (V.3.1.9.2, Dusseldorf, Germany). Two-way Analysis of Variance (ANOVA) was used for the interactions within and between the groups, in order to evaluate the differences between the three groups studied, as well as for the five evaluations during the treatments and the follow-up. The test power was $80 \%, \alpha=0.05$. The sample size was calculated on 171 participants.

\section{Randomisation}

The randomisation will be performed by DFTS, a coauthor of this work, who is not directly linked to the treatments or the evaluation of the participants, by using the Excel 2013 program (Microsoft, USA). The participants will be randomised into blocks of 12 and into 3 groups (4:4:4), designated as A, B and C. Opaque envelopes will be identified by sequence numbers and they will receive a paper containing the information about which treatment will be performed on the participant's abdomen, according to the draw. The sealed envelopes will be safely kept with the researcher who generated the randomisation (DFTS). Before the beginning of the procedures, the researcher responsible for the procedure (LRM, a coauthor of this protocol) will receive each envelope and proceed with the treatment as indicated, according to its allocation group. A team of undergraduate students (previously trained and prepared) is going to be part of the research group and for the treatment or evaluation of the participants. This study will be a double-blind study since the participants will not be aware of the group in which he/she is participating; only the researcher who will perform the procedure will know. The data collection and analyses will be performed by another researcher (MM, a coauthor of this study), who will also be unaware of the allocations.

\section{Intervention}

The abdomen of the participants will be cleaned by using a neutral cleansing soap. They will receive eye protection, using goggles for safety. This will also help with the blindness of the study. PBM will be applied when using abdominal straps as developed by Cosmedical (Mauá, São Paulo, Brazil), following the parameters as described in table 1 . The abdomen strap will be covered with a sheet and that will also help with the blindness of the study. All of the participants will receive $30 \mathrm{~min}$ of $\mathrm{PBM}$, with an abdominal strap containing 4 LED clusters with 72 devices, following the indication of the randomisation, being 57 per group: Group A-RED $660 \pm 10 \mathrm{~nm}$; Group B-INFRARED 830 $\pm 10 \mathrm{~nm}$; Group C-PLACEBO. The treatment will consist of eight sessions that will occur two times a week, totalling 1 month of treatments. The placebo group will use a strap with no light emission, but it will emit the same sounds like that of the active device. In order to increase the oxidation of the free fatty acids, the participants will receive $30 \mathrm{~min}$ of Aussie Current at 4 $\mathrm{kHz}$, modulated at $10 \mathrm{~Hz}, 40-60 \mathrm{~mA}$, for $30 \mathrm{~min}$ (Tensor, 


\begin{tabular}{|c|c|c|}
\hline Parameter & Red LED & Infrared LED \\
\hline Centre wavelength (nm) & 660 & 830 \\
\hline Spectral width (nm) & 20 & 20 \\
\hline Operating mode & Continuous & Continuous \\
\hline $\begin{array}{l}\text { Average radiant power-one } \\
\text { LED }(\mathrm{mW})\end{array}$ & 5 & 5 \\
\hline $\begin{array}{l}\text { Aperture diameter-one LED } \\
(\mathrm{cm})\end{array}$ & 0.3 & 0.3 \\
\hline $\begin{array}{l}\text { Power density at aperture } \\
\left(\mathrm{mW} / \mathrm{cm}^{2}\right)\end{array}$ & 71 & 71 \\
\hline $\begin{array}{l}\text { Beam spot size at target- } \\
\text { one LED }\left(\mathrm{cm}^{2}\right)\end{array}$ & 0.071 & 0.071 \\
\hline Total number of LEDs & 288 & 288 \\
\hline Area irradiated $\left(\mathrm{cm}^{2}\right)$ & 20.4 & 20.4 \\
\hline Irradiance at target $\left(\mathrm{mW} / \mathrm{cm}^{2}\right)$ & 71 & 71 \\
\hline Exposure duration (s) & 1800 & 1800 \\
\hline Radiant exposure (J/cm²) & 127.8 & 127.8 \\
\hline $\begin{array}{l}\text { Energy density at aperture } \\
\left(\mathrm{J} / \mathrm{cm}^{2}\right)\end{array}$ & 127.8 & 127.8 \\
\hline Radiant energy (kJ) & 2.6 & 2.6 \\
\hline Application technique & Contact & Contact \\
\hline $\begin{array}{l}\text { Number and frequency of } \\
\text { treatment sessions }\end{array}$ & $\begin{array}{l}2 \times \text { week for a } \\
\text { month, a total } \\
\text { of } 8 \text { sessions }\end{array}$ & $\begin{array}{l}2 \times \text { week for a } \\
\text { month, } \\
\text { a total of } 8 \\
\text { sessions }\end{array}$ \\
\hline
\end{tabular}

DGM Electronica, Santo André, São Paulo, Brazil). ${ }^{33}$ None of the PBM devices will significantly increase the temperature at the target area, causing no burns or skin damage. No modifications in the intervention will be performed for any reason. However, the participants who withdraw their consent, or the ones who are not assiduous to the sessions, will be removed from the study.

The dosimetric parameters that will be used in this protocol, as presented in table 1, were measured and/or calculated. The centre wavelength and the spectral width of the devices were measured by a Spectrophotometer (USB2000+XR1, Ocean Optics, Florida, USA). The radiant power of one LED was measured by a Power Meter (FieldMaxII-TO, Coherent, Santa Clara, California, USA). The abdominal straps will be composed of 4 LED clusters, having 72 LEDs each (totalling 288 LEDs units), and distributed in a $15 \mathrm{~cm} \times 25 \mathrm{~cm}$ area (375 $\mathrm{cm}^{2}$ each, $1500 \mathrm{~cm}^{2}$ total), see figure 1 . The effective irradiated area will be $20.4 \mathrm{~cm}^{2}$ (288 times the beam spot size at the target). The irradiance at the target was determined by the ratio between the average radiant power $(5 \mathrm{~mW})$ and the beam spot site at the target $\left(0.071 \mathrm{~cm}^{2}\right)$. The radiant exposure was determined by multiplying the irradiance at the target $\left(71 \mathrm{~mW} / \mathrm{cm}^{2}\right)$ by the exposure duration $(1800 \mathrm{~s})$. The radiant energy was calculated by multiplying the average radiant power of one LED ( $5 \mathrm{~mW}$ ) by the total number of LEDs (288) and by the exposure duration (1800 s).
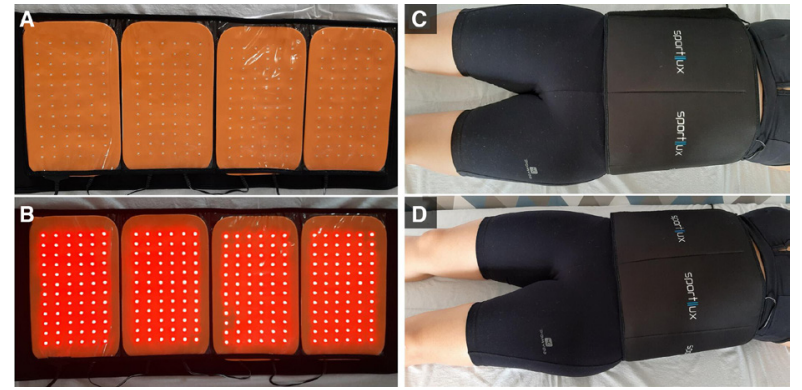

Figure 1 Photobiomodulation (PBM) application. PBM device off $(A)$ and on $(B)$; patient receiving the experimental protocol in dorsal decubitus; 30 min per session (C) and (D).

\section{Outcomes}

The main outcome of this study will be waist circumference reduction. The secondary variables will be anthropometric data, lipid profile, liver function and adipose tissue thickness as measured by ultrasound, changes in the local microcirculation, quality of life and self-esteem. The participants will be evaluated at the same time of the day, at all times throughout the study.

The anthropometric data that will be collected will be body weight, height and BMI, skin-fold thickness and bioimpedance. Blood will be collected for analyses of the lipid profile (total cholesterol, high-density lipoprotein (HDL), Lowdensity lipoprotein (LDL), triglycerides), and liver function (serum glutamic-oxaloacetic transaminase - SGOT, serum glutamic pyruvic transaminase -SGPT). All of this will be processed and analysed at SCS Medicina Diagnóstica, São Caetano do Sul, Brazil (a partner laboratory). The analyses will be performed at four stages of the research, D0, end of the eighth session (D30), 15 days after the last session (FU15), 90 days after the last session (FU90) and 180 days after the last session (FU180).

Abdominal ultrasound will be performed to assess the fat layer thickness, before and after the treatments. For the recording of the local temperature, a technique that is widely used is infrared thermography, using a Compact Thermal Camera (C2, FLIR Systems, Oregon, USA). The thermal camera, by means of infrared emission from the body, or from the material analysed, has the ability to calculate the temperature of a given surface. It is possible through this method that the study will infer the changes in local microcirculation. ${ }^{34}$

The quality of life questionnaire, 'The WHO Quality of Life WHOQOL-BREF', ${ }^{35}$ as well as the Body Shape Questionnaire, 'BSQ-34 Self-Image Scale', ${ }^{36}$ will be used for the participants. These questionnaires have been translated and submitted to cross-cultural adaption into Brazilian Portuguese. ${ }^{37} 38$ The Brazilian Portuguese version of these questionnaires will be applied by MM. The questionnaires will take around $20 \mathrm{~min}$ to be completed. The quality of life and the self-image questionnaires will be applied at D0-and again at the end of the last session (D30). The flow chart of the study is presented in figure 2.

Adverse events will be collected during the treatment sessions and they will be reported to the regulatory agency and again, 


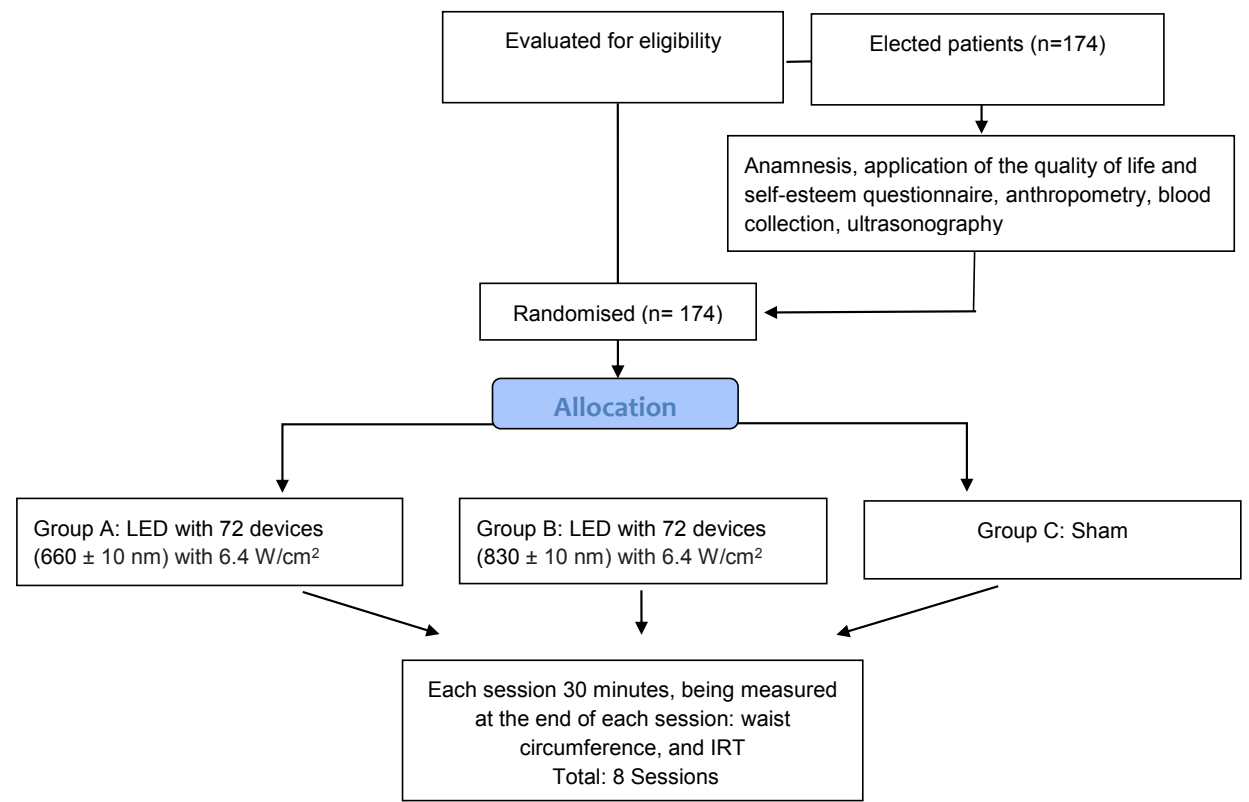

Analysis at the end of treatment

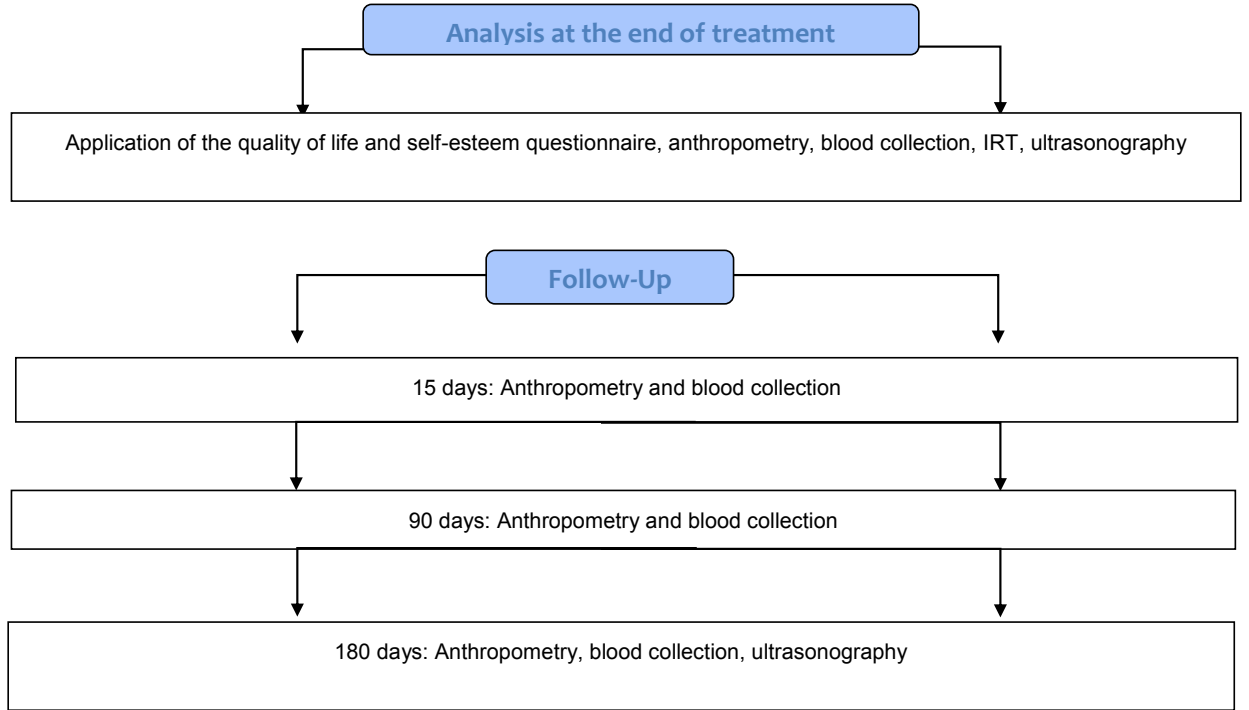

Figure 2 Flow chart describing the study design, the sample composition and the experimental protocol. IRT, infrared thermography.

at the final publication of the results. Since the participants are enrolled and randomised, the investigators will make efforts to keep the participants together during the follow-up, by making phone calls, email/WhatsApp contact with the patients and with relevant instructions regarding healthcare and beauty.

As a strategy to improve adherence, at each session, the participant will schedule the next visit and receive a card, with some instructions regarding preparation for the evaluation day, and the appointment date of the next visit. When a participant misses a session, he/she will receive a phone call in order to reschedule the missed session.

\section{Data analysis plan}

The data that will be collected from this study will only be administered by the principal investigators (the authors of this document). Since the study will be of short duration and with known minimal risks, this trial will not need a formal data monitoring committee. After the data collection, the data will be organised using Microsoft Office Excel 2007 by DFTS (coauthor of this protocol), and then stored on 'a protected by password' computer at the university. The data will be analysed by descriptive and inferential statistics, and then compiled into tables and/or graphs, using SPSS V.24.0. For testing the normality of the data, the Shapiro-Wilk test will be performed. If the data show a non-parametric behaviour, a mathematical function will be used in order to normalise the data. Two-way ANOVA tests, followed by the Bonferroni posttest, will be performed, in order to compare the treatments along with the time points being evaluated. Some parameters 
may affect the results of the therapy and they are going to be analysed as co-variables; for example, skin phototype by the Fitzpatrick scale; the stage of the menstrual cycle; total cholesterol and triglycerides (altered or not). $\alpha<0.05$ will be considered the level of significance for all of the tests used. Since this trial is part of a $\mathrm{PhD}$ thesis study, an interim analysis will be performed for the qualification examination, and this can be used at trial adaptations, such as for a sample size re-estimation, or for stopping the trial. The trial protocol and the full study report will be fully available at the end of the study after the manuscript of the results has been published. At the end of the study, the participants from the placebo group who received the treatment will experience no adverse effects and they will have received the most effective treatment.

\section{DISCUSSION}

Studies have shown that lasers used in PBM typically operate at powers of $100 \mathrm{~mW}$ or less. They can produce energy in the visible spectrum, wavelengths $400-700 \mathrm{~nm}$ and near the infrared regions, $700-1500 \mathrm{~nm}$. Light penetration in the soft tissues is known to be directly related to wavelength; that is, the longer the wavelength, the greater the penetration. The reports on PBM for reducing local adiposity include the use of green $(532 \mathrm{~nm})$, red (635 and $660 \mathrm{~nm})$ and infrared (1060 and $2940 \mathrm{~nm}$ ). However, there is no comparison available regarding the best wavelength for this purpose. ${ }^{18-22} 28$ Based on the localisation of fat tissue (more profound when compared with epidermis and dermis), this study will choose red and infrared light for the comparisons. The use of the same dosimetry, at these different wavelengths, will allow for the evaluation of the most suitable wavelength for body contouring.

Since the waist circumference measurements will not discriminate against the factors underlying the volume modifications, a placebo group will be included. This will allow for the measurement of the differences in waist circumference, due to daily habits or hormonal variations, as in the menstrual cycle of women. The measurements of the skin folds and bioimpedance will complement the evaluation in terms of body fat. At each evaluation (before the treatment and the follow-up sessions), 3 days of food records and physical activity levels will be measured.

Gender may also affect the results. Sexual dimorphism in adipogenesis is already known, as well as sex hormones in the white adipose tissue function and in adipose metabolism. ${ }^{39-41}$ If the sample consists of a huge difference in men and women, this factor cannot be considered in the statistical analysis.

The development of a non-invasive protocol for PBM, together with an Aussie current for the reduction of adiposity, may present an important novel tool, for the reduction of health risk problems, as well as for increasing an individual's self-esteem.

\section{ETHICS AND DISSEMINATION}

The Ethics Committee of the Nove de Julho University, São Paulo, Brazil, approved the modified version of this project and the Patient Informed Consent Form under No. 3414146 on 26 June 2019, according to the guidelines of the Brazilian National Ethics Committee. The protocol of this study has already been registered in the Brazilian Registry of Clinical Trials, being first registered on 8 November 2018, and modified on 28 August 2019, providing full public access to the protocol information, including all items from the WHO Trial Registration Data Set. MM and DFTS will be the data curators, with the data stored on 'a protected by password' computer at the university. The results acquired within this project will be presented in conferences and published in a journal in the related field. The authorship of the results paper and the conference abstracts will include the authors of this protocol, together with other researchers who may contribute to the procedures or to the analysis of the data. Any modifications of this protocol will require a formal amendment and they will be approved by the Ethics Committee of the Nove de Julho University. The modifications will be properly reported and justified in the manuscript for the publication of the results. The main results obtained will be sent to the participants by mail.

Acknowledgements The authors would like to thank the Nove de Julho University (UNINOVE), São Paulo, Brazil, for the availability of its laboratories, the company Cosmedical, for the development of the equipment for PBM and the SCS Medicina Diagnóstica Laboratory, São Caetano do Sul, Brazil, for their partnership in the analyses of the laboratory tests.

Contributors MM, LR-M, DFTS and CP designed the study. MM and LR-M will conduct the experiments and will be making the data acquisitions. DFTS and CP will perform data analysis and interpretation. MM and LR-M drafted the work, while $\mathrm{CP}$ and DFTS revised it critically for important intellectual content. All of the authors approved the final version of the manuscript.

Funding The authors have not declared a specific grant for this research from any funding agency in the public, commercial or not-for-profit sectors.

Competing interests None declared.

Patient consent for publication Obtained.

Provenance and peer review Not commissioned; externally peer reviewed.

Open access This is an open access article distributed in accordance with the Creative Commons Attribution Non Commercial (CC BY-NC 4.0) license, which permits others to distribute, remix, adapt, build upon this work non-commercially, and license their derivative works on different terms, provided the original work is properly cited, appropriate credit is given, any changes made indicated, and the use is non-commercial. See: http://creativecommons.org/licenses/by-nc/4.0/.

\section{ORCID iDs}

Daniela Fátima Teixeira Silva http://orcid.org/0000-0002-7228-6146

Christiane Pavani http://orcid.org/0000-0001-8275-7370

\section{REFERENCES}

1 Silva Figueiredo P, Carla Inada A, Marcelino G, et al. Fatty acids consumption: the role metabolic aspects involved in obesity and its associated disorders. Nutrients 2017;9:1158-32.

2 Landecho MF, Tuero C, Valentí V, et al. Relevance of leptin and other adipokines in obesity-associated cardiovascular risk. Nutrients 2019;11:2664-16.

3 Kong Y, Zhang S, Wu R, et al. New insights into different adipokines in linking the pathophysiology of obesity and psoriasis. Lipids Health Dis 2019:1-12.

4 Jiménez Flores P, Jiménez Cruz A, Bacardi Gascón M. Body-image dissatisfaction in children and adolescents: a systematic review. Nutr Hosp 2017;34:479-89.

5 Weinberger N-A, Kersting A, Riedel-Heller SG, et al. Body Dissatisfaction in individuals with obesity compared to normal-weight 
individuals: a systematic review and meta-analysis. Obes Facts 2016;9:424-41.

6 Kouris A, Platsidaki E, Christodoulou C, et al. Patients' self-esteem before and after chemical peeling procedure. J Cosmet Laser Ther 2018;20:220-2.

7 Stundzaite-Barsauskiene G, Tutkuviene J, Barkus A, et al. Facial perception, self-esteem and psychosocial well-being in patients after nasal surgery due to trauma, cancer, and aesthetic needs (cluster analysis of multiple interrelations). Ann Hum Biol 2019;21:1-16.

8 Ribeiro F, Steiner D. Quality of life before and after cosmetic procedures on the face: a cross-sectional study in a public service. $J$ Cosmet Dermatol 2018;17:688-92.

9 Bensoussan J-C, Bolton MA, Pi S, et al. Quality of life before and after cosmetic surgery. CNS Spectr 2014;19:282-92.

10 Appleton SE, Ngan A, Kent B, et al. Risk factors influencing transfusion rates in DIEP flap breast reconstruction. Plast Reconstr Surg 2011;127:1773-82.

11 Lievain L, Aktouf A, Auquit-Auckbur I, et al. [Abdominoplasty complications: particularities of the post-bariatric patients within a 238 patients series]. Ann Chir Plast Esthet 2015;60:26-34.

12 Sterodimas A, Boriani F, Nicaretta B, et al. Revision Abdominoplasty with truncal Liposculpting: a 10-year experience. Aesthetic Plast Surg 2019;43:155-62.

13 Al Dujaili Z, Karcher C, Henry M, et al. Fat reduction: complications and management. J Am Acad Dermatol 2018;79:197-205.

14 Krueger N, Mai SV, Luebberding S, et al. Cryolipolysis for noninvasive body contouring: clinical efficacy and patient satisfaction. Clin Cosmet Investig Dermatol 2014;7:201-5.

15 Neira R, Arroyave J, Ramirez H, et al. Fat liquefaction: effect of low-level laser energy on adipose tissue. Plast Reconstr Surg 2002;110:912-22.

16 Karu T. Mitochondrial mechanisms of photobiomodulation in context of new data about multiple roles of ATP. Photomed Laser Surg 2010;28:159-60.

17 Feng J, Zhang Y, Xing D. Low-power laser irradiation (LPLI) promotes VEGF expression and vascular endothelial cell proliferation through the activation of ERK/Sp1 pathway. Cell Signal 2012;24:1116-25.

18 Croghan IT, Hurt RT, Schroeder DR, et al. Low-level laser therapy for weight reduction: a randomized pilot study. Lasers Med Sci 2020;35:663-75.

19 Thornfeldt CR, Thaxton PM, Hornfeldt CS. A six-week low-level laser therapy protocol is effective for reducing waist, hip, thigh, and upper abdomen circumference. J Clin Aesthet Dermatol 2016;9:31-5.

20 McRae E, Boris J. Independent evaluation of low-level laser therapy at $635 \mathrm{~nm}$ for non-invasive body contouring of the waist, hips, and thighs. Lasers Surg Med 2013;45:1-7.

21 Mazzoni D, Lin MJ, Dubin DP, et al. Review of non-invasive body contouring devices for fat reduction, skin tightening and muscle definition. Australas J Dermatol 2019;60:278-83.

22 Nestor MS, Newburger J, Zarraga MB. Body contouring using 635nm low level laser therapy. Semin Cutan Med Surg 2013;32:35-40.

23 Jankowski M, Gawrych M, Adamska U, et al. Low-level laser therapy (LLLT) does not reduce subcutaneous adipose tissue by local adipocyte injury but rather by modulation of systemic lipid metabolism. Lasers Med Sci 2017;32:475-9.

24 Avci P, Nyame TT, Gupta GK, et al. Low-level laser therapy for fat layer reduction: a comprehensive review. Lasers Surg Med 2013;45:349-57.
25 da Silveira Campos RM, Dâmaso AR, Masquio DCL, et al. Low-Level laser therapy (LLLT) associated with aerobic plus resistance training to improve inflammatory biomarkers in obese adults. Lasers Med Sci 2015;30:1553-63.

26 Duarte FO, Sene-Fiorese M, de Aquino Junior AE, et al. Can low-level laser therapy (LLLT) associated with an aerobic plus resistance training change the cardiometabolic risk in obese women? A placebo-controlled clinical trial. J Photochem Photobiol B 2015;153:103-10.

27 Jackson RF, Dedo DD, Roche GC, et al. Low-level laser therapy as a non-invasive approach for body contouring: a randomized, controlled study. Lasers Surg Med 2009;41:799-809.

28 Vas K, Besenyi Z, Urbán S, et al. Efficacy and safety of long pulse 1064 and $2940 \mathrm{~nm}$ lasers in noninvasive lipolysis and skin tightening. $J$ Biophotonics 2019;12:1-8.

29 Nestor MS, Zarraga MB, Park H. Effect of $635 \mathrm{~nm}$ low-level laser therapy on upper arm circumference reduction: a double-blind, randomized, sham-controlled trial. J Clin Aesthet Dermatol 2012;5:42-8.

30 Caruso-Davis MK, Guillot TS, Podichetty VK, et al. Efficacy of lowlevel laser therapy for body contouring and spot fat reduction. Obes Surg 2011;21:722-9.

31 Grosset J-F, Crowe L, De Vito G, et al. Comparative effect of a 1 $\mathrm{H}$ session of electrical muscle stimulation and walking activity on energy expenditure and substrate oxidation in obese subjects. Appl Physiol Nutr Metab 2013;38:57-65.

32 Savoia A, Landi S, Vannini F, et al. Low-level laser therapy and vibration therapy for the treatment of localized adiposity and fibrous cellulite. Dermatol Ther 2013;3:41-52.

33 Canela VC, Crivelaro CN, Ferla LZ, et al. Synergistic effects of combined therapy: nonfocused ultrasound plus Aussie current for noninvasive body contouring. Clin Cosmet Investig Dermatol 2018;11:203-12.

34 Neubauer-Geryk J, Hoffmann M, Wielicka M, et al. Current methods for the assessment of skin microcirculation: Part 1. Postepy Dermatol Alergol 2019;36:247-54.

35 THE WHOQOL GROUP. Development of the world Health organization WHOQOL-BREF quality of life assessment. The WHOQOL group. Psychol Med 1998;28:551-8.

36 Cooper PJ, Taylor MJ, Cooper Z, et al. The development and validation of the body shape questionnaire. Int $J$ Eat Disord 1987;6:485-94.

37 Di Pietro M, Silveira DXda, Di PM. Internal validity, dimensionality and performance of the body shape questionnaire in a group of Brazilian college students. Braz J Psychiatry 2009;31:21-4.

38 Fleck MP, Louzada S, Xavier M, et al. [Application of the Portuguese version of the abbreviated instrument of quality life WHOQOL-bref]. Rev Saude Publica 2000;34:178-83.

39 Savva C, Korach-André M. Estrogen receptor beta (ER $\beta$ ) regulation of lipid Homeostasis-Does sex matter? Metabolites 2020;10:116.

40 White UA, Tchoukalova YD. Sex dimorphism and depot differences in adipose tissue function. Biochim Biophys Acta 2014;1842:377-92.

41 Fuller-Jackson J-P, Dordevic AL, Clarke IJ, et al. Effect of sex and sex steroids on brown adipose tissue heat production in humans. Eur $J$ Endocrinol 2020:1-36. 